\title{
Partikelmessung an hochkonzentrierten Dispersionen nach dem Ultraschall-Reflexionsprinzip
}

\author{
Robert Weser, Benno Wessely \\ Technische Universität Dresden, Institut für Verfahrenstechnik und Umwelttechnik, Arbeitsgruppe \\ Mechanische Verfahrenstechnik, 01062 Dresden \\ Sebastian Wöckel, Ulrike Hempel \\ Institut für Automation und Kommunikation (ifak) e.V. Magdeburg, Werner-Heisenberg-Str. 1, \\ 39106 Magdeburg
}

\section{Motivation}

Für eine Vielzahl von Anwendungen in der Verfahrenstechnik, Biotechnologie oder Lebensmittelindustrie ist die Charakterisierung der Feststoffphase in hochkonzentrierten Dispersionen anhand der Partikelgrößenverteilung und -konzentration von wesentlicher Bedeutung. Die online bzw. inline gewonnenen Daten können dazu beitragen, die verschiedenen verfahrenstechnischen Prozesse optimal zu steuern, Produktqualitäten gezielt zu steigern oder Anlagenverfügbarkeiten zu erhöhen. Exemplarische Vorgänge wie die Emulgierung und (Nass-)Zerkleinerung finden sich in einer Vielzahl industriell relevanter Prozesse, wie beispielsweise der Herstellung von Lacken und Farben oder der Aufbereitung von Erz- und Klärschlämmen. Während vor allem bei einem hohen Partikelanteil und der damit verbundenen Opazität der Einsatz etablierter optischer Methoden begrenzt ist, können auf Ultraschall (US) basierende Messmethoden einen Vorteil bei der prozessnahen oder Inline-Charakterisierung bieten. Häufig angewandtes Untersuchungsverfahren ist die Extinktionsmessung, bei der das Stoffsystem durchschallt und die sender- und empfängerseitige Intensität spektral verglichen wird. Daraus lassen sich die für die Partikelcharakterisierung relevanten Informationen ableiten. Aufgrund der erheblichen Schalldämpfung ist es insbesondere bei hoch konzentrierten Stoffsystemen erforderlich, den Abstand zwischen Sende- und Empfangswandler hinreichend klein zu gestalten $(0,5 \mathrm{~mm}-2 \mathrm{~mm})$, um ein noch auswertbares Messsignal zu erhalten. Dies führt bei Inline-Anwendungen häufig zu Verstopfungen innerhalb der Messstrecke (vor allem beim Vorhandensein einzelner großer Feststoffpartikel oder pastöser Medien), was die Zuverlässigkeit und die Akzeptanz der Messeinrichtung bei den Anwendern einschränkt.

Ein Erfolg versprechender Ansatz zur Problemlösung wird darin gesehen, die vom Stoffsystem gestreuten (statt transmittierten) Schallanteile zu analysieren. Äquivalent zur Extinktionsanordnung ermöglicht dies Aussagen zur dispersen Phase, ohne das Risiko der kritischen Verstopfung der Messstrecke einzugehen (Vermeidung des Messspaltes). Im Beitrag wird das Konzept eines auf Ultraschall-Rückstreuung basierenden Messverfahrens zur Partikelcharakterisierung in hochkonzentrierten Dispersionen vorgestellt (vgl. Bild 1). Kerngedanke des experimentellen Aufbaus ist der Einsatz breitbandiger Sende-/Empfangs-wandler, die hinsichtlich einer hohen Sendeleistung, eines der Eindringtiefe entsprechenden Messfensters und hoher Empfangssensitivität optimal aufeinander abgestimmt sind. Auf diese Weise soll der von den Partikeln gestreute Schallanteil erfasst und hinsichtlich der Intensität, der Laufzeit (entspricht der Eindringtiefe) und der Schallfrequenz analysiert werden.

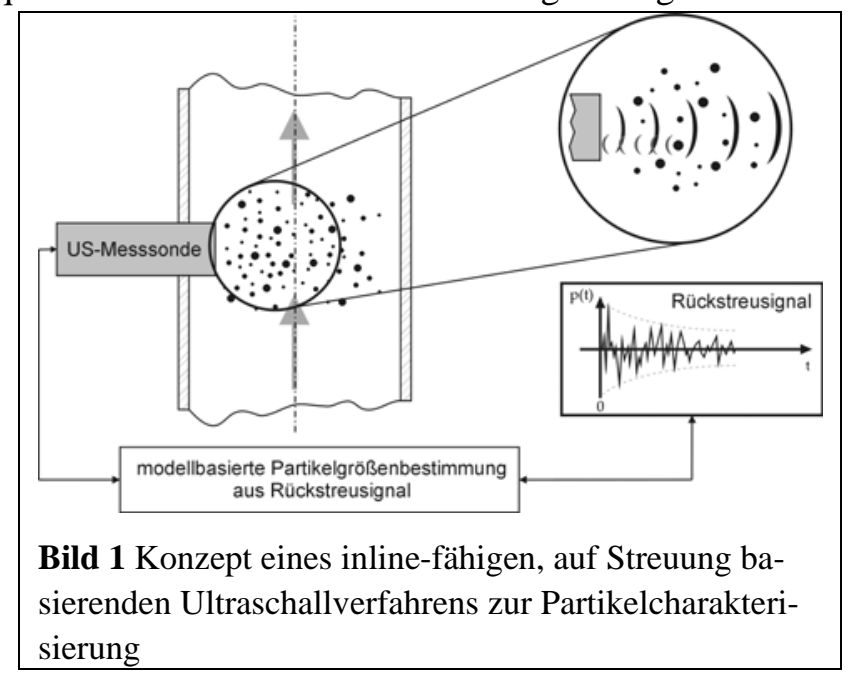

\section{Messaufbau}

Bild 1 verdeutlicht schematisch den grundlegenden Aufbau des auf Ultraschallstreuung basierenden Verfahrens. Im Hinblick auf eine kostenoptimierte Lösung wird kommerziell verfügbare Hardware (Schallwandler, Elektronik zur Signalgenerierung und -verarbeitung) der Neuentwicklung einer Spezialhardware vorgezogen. 
Bei der Anordnung der US-Wandler werden mehrere Konzepte verfolgt. Neben der klassischen Reflexion (ein Sende-/Empfangswandler mit Vorlaufstrecke, direkte Rückstreuung) sollen auch die unter definierten Winkeln gestreuten Schallanteile (getrenntes US-Wandlerpaar) analysiert werden. Die Bereitstellung definierter Messbedingungen ist für die experimentellen Untersuchungen von besonderer Bedeutung. So wird die Dispersion während der Messung temperiert und der Dispergierzustand sowie die Partikelkonzentration mittels Laboranalytik überwacht. Weiterhin muss durch eine geeignete Strömungsführung vor der USMesssonde sichergestellt werden, dass das Partikelsystem homogen im Messvolumen verteilt ist.

\section{Reflexionsmessung nach dem Puls-Echo-Verfahren}

Zunächst wurde ein vergleichsweise einfacher Messaufbau zur Erfassung von US-Echosignalen mit einem Sende-/Empfangswandler und konventioneller Impulsanregung umgesetzt (vgl. Bild 2). Eine wasserbefüllte Vorlaufstrecke schützt die aktive Wandleroberfläche vor auftretender Abrasion durch die Partikel. Diese direkte US-Rückstreumessung liefert nach Anregung des Schallwandlers ein Echosignal (Amplitude $p$ in Abhängigkeit von der Laufzeit $t$ ), das neben den Reflexionspeaks des Vorlaufstreckenfensters ebenfalls die Rückstreusignale der Partikel enthält (vgl. Bild 2). Allerdings sind die Partikelsignale aufgrund ihrer geringen Amplitude vor dem Hintergrund der Fensterreflexionen schlecht zu erkennen bzw. davon zu unterscheiden. Daher wurde anstelle der Echoamplitude deren Standardabweichung in Abhängigkeit von der Laufzeit ausgewertet. Nach Erfassung von $N$ Einzelmessungen (Echosignal infolge eines ausgesendeten Impulses) wird zu jeder Laufzeit $t$ die Standardabweichung der jeweils $N$ Amplitudenwerte bestimmt. Die Fensterreflexionen (und deren Mehrfachreflexionen) stellen statische Signalanteile im Echozeitsignal dar und liefern damit keinen Anteil zur derart bestimmten Standardabweichung. Lediglich die zeitlich veränderlichen Partikelsignale (infolge der Bewegung der Partikel durch das Messvolumen) erzeugen eine relevante Standardabweichung.

Beim Durchlaufen der Dispersion werden sowohl die emittierte als auch die an den Partikeln gestreuten Schallwellen in Abhängigkeit von der zurückgelegten Wegstrecke gedämpft. Die vom Schallwandler detek-

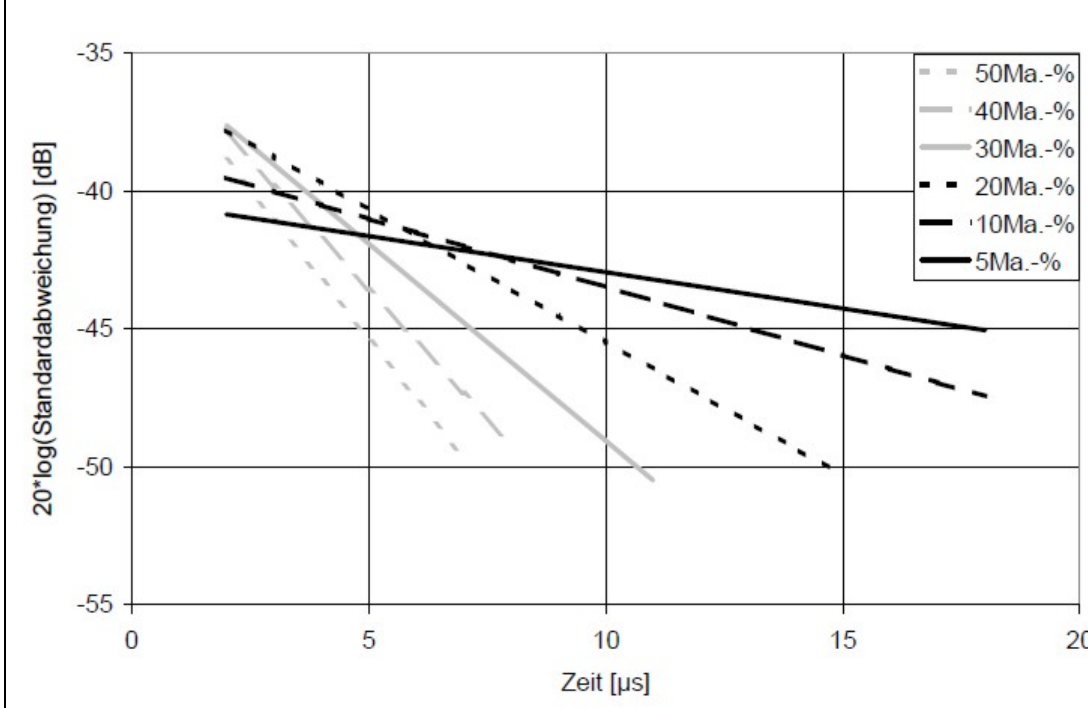

Bild 3 Logarithmische Standardabweichung (über 100 Einzelmessungen) in Abhängigkeit von der Laufzeit für unterschiedlichen Partikelkonzentrationen an $\mathrm{SiO}_{2}$-Partikel in Wasser, Darstellung der linearen Fit-Funktionen tierbare Echosignalamplitude verringert sich demnach mit zunehmender Laufzeit - gleiches gilt für die Standardabweichung. Die Schallabschwächung wächst exponentiell mit der durchschallten Wegstrecke bzw. der Laufzeit an und wird u. a. von der Partikelgröße und -konzentration bestimmt [1], [2]. Dieser Effekt konnte in einer ersten Messreihe nachgestellt werden. Dazu wurde die direkte USRückstreuung aus einer Suspension von $\mathrm{SiO}_{2}$-Partikeln (Dichte: 2,35 g/ $\mathrm{cm}^{3}$, Partikelgrößenbereich kleiner $20 \mu \mathrm{m}$ ) in Wasser aufgenommen. Die Partikelkonzentration wurde stufenweise auf bis zu 50 Ma.-\% erhöht und die entspre- 
chenden Suspensionsansätze zusätzlich mit einem US-Spektrometer [3] vermessen. Im Ergebnis der Messungen zeigt Bild 3 den zeitlichen Verlauf der Standardabweichung für die verschiedenen Partikelkonzentrationen. Die Standardabweichung ist logarithmiert (in Dezibel) aufgetragen, da der exponentielle Einfluss der Schalldämpfung auf diese Weise linearisiert dargestellt werden kann. Mit zunehmender Partikelkonzentration fallen die an die Kurvenverläufe angepassten Geraden (Fit-Funktionen) stärker ab, gleichzeitig steigt die Schalldämpfung an. Der Betrag der Geradenanstiege entspricht der (laufzeitabhängigen) Schalldämpfung

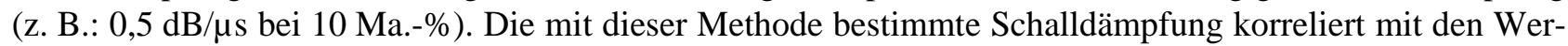
ten der Referenzmessung (Tafel 1).

Tafel 1 Schalldämpfung in Abhängigkeit von der Partikelkonzentration, Vergleich mit Referenzmesswerten des USSpektrometers (DT1200), Schallgeschwindigkeit: $1460 \ldots 1500 \mathrm{~m} / \mathrm{s}$ (abhängig von Partikelkonzentration), $f=4,5 \mathrm{MHz}$

\begin{tabular}{c|c|c|c}
\hline $\begin{array}{c}\text { Partikelkonzentration } \\
{[\text { Ma.-\%] }}\end{array}$ & $\begin{array}{c}\text { Schalldämpfung } \\
{[\mathrm{dB} / \mu \mathrm{ss}]}\end{array}$ & $\begin{array}{c}\text { Schalldämpfung } \\
{[\mathrm{dB} / \mathrm{cm} / \mathrm{MHz}]}\end{array}$ & $\begin{array}{c}\text { Schalldämpfung DT1200 } \\
{[\mathrm{dB} / \mathrm{cm} / \mathrm{MHz}]}\end{array}$ \\
\hline 10 & 0,5 & 0,75 & 0,63 \\
\hline 20 & 0,96 & 1,44 & 1,36 \\
\hline 30 & 1,43 & 2,16 & 2,12 \\
\hline 40 & 1,9 & 2,89 & 2,84 \\
\hline 50 & 2,16 & 3,31 & 3,48 \\
\hline
\end{tabular}

\section{Erhöhung der Schallleistung durch Signalcodierung}

Die Schallstreuung, d.h. die messbare reflektierte Leistung $P_{\text {OUT }}(1)$, ist abhängig vom Material der Partikel $(\Gamma)$ und deren Geometrie $(\sigma)$ in Relation zur Anregungsfrequenz $f$. Bild 4a zeigt ein vereinfachtes Modell der winkelabhängigen Schallstreuung an Einzelpartikeln mit dem sich Gl. 1 zur Bewertung der Leistungsfähigkeit des Streumessverfahrens ableiten lässt. Ein bewegtes Partikel erzeugt im Sensitivitätsbereich der Schallwandler (Bild 4b) ein in Bild 4c dargestelltes Streusignal, an dem sich Geschwindigkeit und Streuleistung $P_{\text {OUT }}$ des Partikels experimentell bestimmen lassen.

$$
\frac{P_{\text {OUT }}}{P_{I N}}=\frac{4 k_{P}}{\pi d_{W}^{2}} \cdot \frac{\pi d_{P}^{2}}{4} \cdot \sigma \cdot \Gamma \cdot \frac{1}{4 \pi \mathrm{S}^{2}} \cdot \frac{\pi d_{W}^{2}}{4} k_{P}
$$

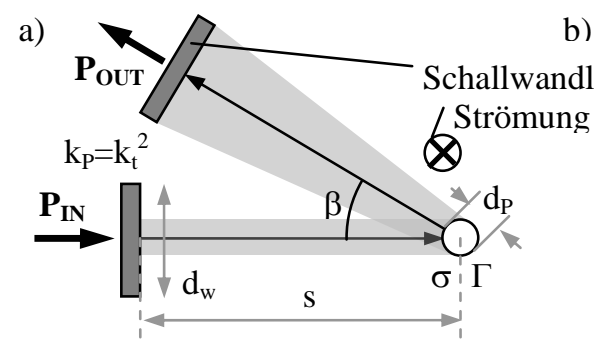

b)
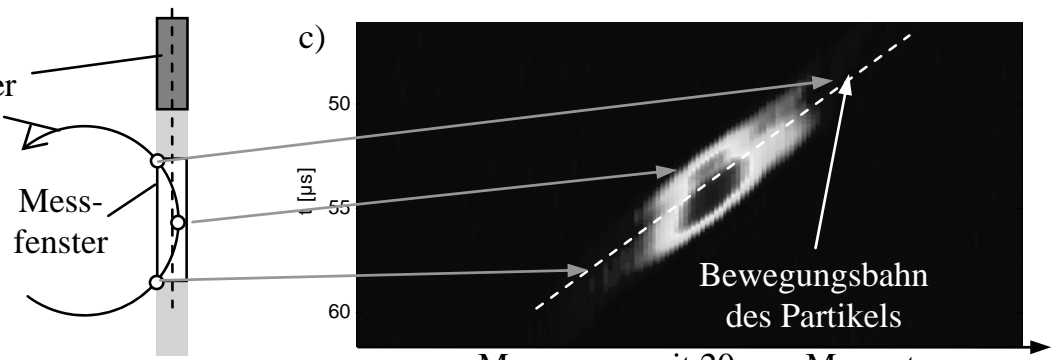

Messungen mit $20 \mu \mathrm{s}$ - Messrate

Bild 4 a) Schematische Darstellung der Streumessung ( $\Gamma$ - Reflektivität des Partikels, $\sigma$ - winkelabhängige Streufunktion, $k_{P}$ - Koppelfaktor, $d$ - Durchmesser, $s$ - Weg), b) Sicht auf Wandler, Messfenster [15 us] und Partikelbahn, c) Streusignal eines sich durch das Messfenster bewegenden Partikels $d=200 \mu \mathrm{m}$

Mit zunehmender Partikelanzahl nimmt die Mehrfachstreuung zu und verringert das messbare Einzelstreusignal nach Gl. 1 zusätzlich. Folglich muss zur Erfassung der Streuung mit repräsentativer Eindringtiefe in das Messvolumen eine höhere Schallleistung $P_{I N}$ in die Suspension eingebracht werden. Für eine hohe Ortsauflösung ist ferner ein Impuls kurzer Zeitdauer $T_{0}$ notwendig (vgl. Bild 5a). Alternativ zu den üblicherweise verwendeten Kurzzeitimpulsen ist insbesondere zur Erhöhung der Leistung die Verwendung von codierten Signalfolgen oder Pseudorauschsignalen (MLBS) möglich (Bild 5b) [4]. Mit diesen lässt sich die Energie des Sendesignals auf das gesamte zeitliche Messfenster $T$ verteilen. Hierdurch verringert sich das Verhältnis von Maximalamplitude zu Effektivwert (Crest-Faktor) deutlich und ermöglicht die Verwendung geringerer elektrischer Spannungen $U_{T}$. Das Frequenzspektrum und die Korrelation der Streusignale für Impulsanregung und codierte Signalfolge sind identisch und stellen die Impulsantwort des Partikelsystems dar [5]. Am Beispiel einer Streumessung unter $90^{\circ}$ mit getrennten Sende- und Empfangswandlern sind in Bild 5 (unten) die Signale von bewegten Einzelpartikeln (500-750) $\mu \mathrm{m}$ aus Polymer im Korrelationsraum dargestellt. Äquivalent lässt sich im Korrelationsraum eine effektive Streudämpfung anhand der Standardabweichung oder 
Amplitude ableiten (s. o.). Voraussetzung zur Verwendung dieses Ansatzes ist ein lineares System, dessen Materialeigenschaften nicht von der Amplitude des eingeprägten akustischen Druckfeldes abhängen.

a)

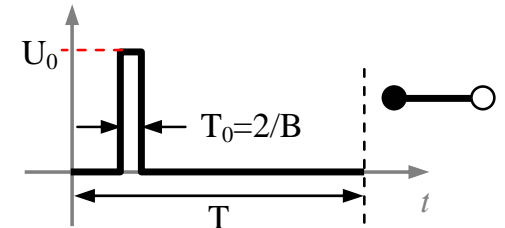

$\bar{P}_{\text {pulse }}=\frac{1}{T} \int_{T} u^{2}(t) d t=U_{0}{ }^{2} \frac{T_{0}}{T}$

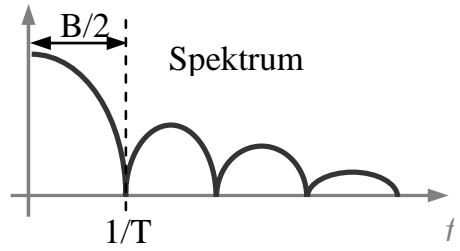

$\bar{P}_{\text {pulse }}^{1 / \mathrm{T}}=\bar{P}_{M L B S}: U_{T}=U_{0} \sqrt{\frac{T_{0}}{T}}$
$T_{0}=0,4 \mu \mathrm{s} \quad T=20 \mu \mathrm{s}$ b) $\uparrow \mathrm{U}_{\mathrm{T}}$

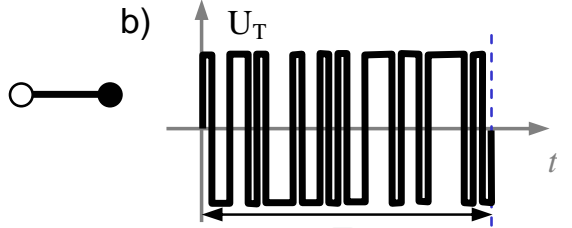

$\bar{P}_{M L B S}=\frac{1}{T} \int_{T}^{T} u^{2}(t) d t=U_{T}{ }^{2}$
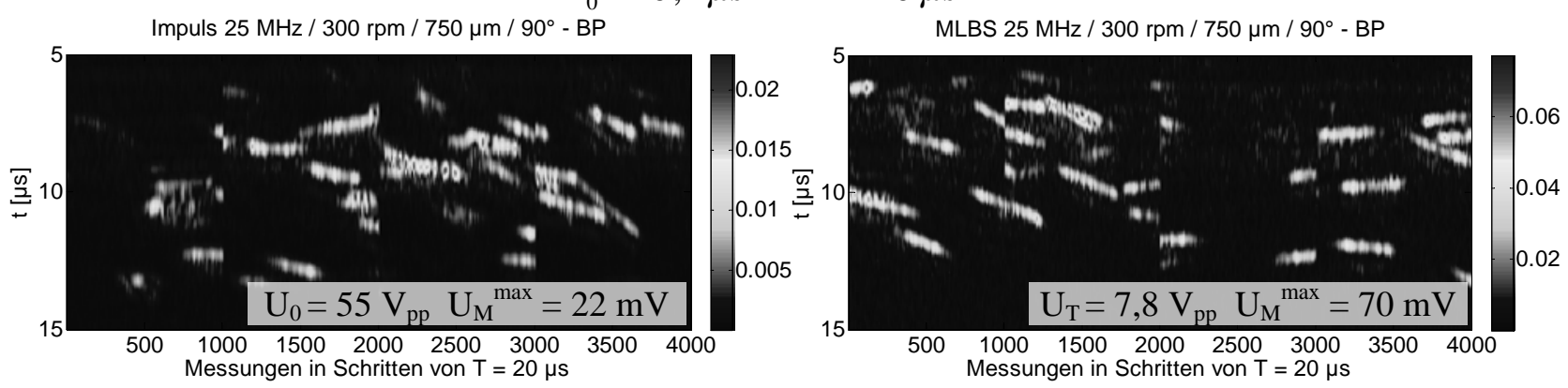

Bild 5 Einhüllende des messbaren Streusignals $U_{M}$ im Korrelationraum für $\beta=90^{\circ}$, Einzelstreuungen eines $25 \mathrm{MHz}-$ Breitbandsignals an strömenden Polymerpartikeln (500-750) $\mu \mathrm{m}$ in Wasser für verschiedene Anregungssignale gleicher Leistung $P_{\text {pulse }}=P_{\text {MLBS }}$. a) Impuls, b) codierte Signalfolge jeweils gemischt mit einem Sinussignal entsprechend der Mittenfrequenz der Ultraschallwandler $(f=25 \mathrm{MHz})$.

\section{Zusammenfassung}

Die Auswertung der laufzeitabhängigen Standardabweichung erlaubt die Bestimmung der US-Dämpfung ohne limitierenden Messspalt, wobei die gefundenen Werte mit jenen des US-Spektrometers gut übereinstimmen. Eine spektrale Analyse der gestreuten Schallsignale steht im Fokus zukünftiger Arbeiten.

Die Verteilung der Energie auf das gesamte Messfenster durch Verwendung codierter Signale begünstigt die Streumessung in Suspensionen mit hoher Partikeldichte (>50 Ma.-\%) und Medien hoher Dämpfung [6]. Die eingebrachte Schalleistung bei konstanter Spannungsamplitude ist um den Faktor des Tastverhältnisses $\mathrm{T} / \mathrm{T}_{0}$ höher als bei einem Kurzzeitimpuls äquivalenter Bandbreite und Ortsauflösung.

\section{Förderung}

Das IGF-Vorhaben 16681 BR / 1 der Forschungsvereinigung Forschungsgesellschaft für Messtechnik, Sensorik und Medizintechnik e.V. Dresden - fms, Theodor-Heuss-Allee 25, 60486 Frankfurt am Main wurde über die AiF im Rahmen des Programms zur Förderung der industriellen Gemeinschaftsforschung und entwicklung (IGF) vom Bundesministerium für Wirtschaft und Technologie aufgrund eines Beschlusses des Deutschen Bundestages gefördert.

Für die Entwicklung des Messaufbaus und die Erstellung der umfangreichen Messdaten zur Partikelstreuung danken wir Herrn Dipl.-Ing. H. Arndt (ifak).

\section{Literatur}

[1] R. Millner: Wissensspeicher Ultraschalltechnik. 1. Aufl. Leipzig: VEB Fachbuchverlag 1987.

[2] A.S. Dukhin, P.J. Goetz: Ultrasound for Characterizing Colloids, Elsevier, 2002.

[3] DT100 / DT1200 Ultraschallspektrometer, Dispersion Technology, Inc., http://dispersion.com

[4] H. Alrutz: Über die Anwendung von Pseudorauschfolgen zur Messung an linearen Übertragungssystemen. Dissertation, Georg-August-Universität Göttingen, 1983.

[5] J. Sachs, R. Thomä: Vergleichende Untersuchungen zum Einsatz ausgewählter Testsignale in der akustischen Materialprüfung, DAGA 1996, Saarbrücken, 1996.

[6] A. Nowicki, I. Secomski, J.L. Trots: Extending penetration depth using coded ultrasonography. Bulletin of the polish Academy of Sciences. Band 52, Nr. 3, 2004. 